\title{
Crystal structure of 6-(4-difluoromethoxy-3-methoxyphenyl)-3(2H)- pyridazinone, $\mathrm{C}_{12} \mathrm{H}_{10} \mathrm{~F}_{2} \mathrm{~N}_{2} \mathrm{O}_{3}$
}

\author{
C. R. Doyle ${ }^{\mathrm{I}}$, M. D. Zimmerman', M. Chruszcz', M. Cymborowski', A. Gawlicka-Chruszcz ${ }^{\mathrm{II}}$ and W. Minor*,I \\ I University of Virginia, Department of Molecular Physiology and Biological Physics, 1300 Jefferson Park Avenue, Charlottesville, VA 22908, USA \\ II HKL Research, Inc., 310 Old Ivy Way, Charlottesville, VA 22903, USA
}

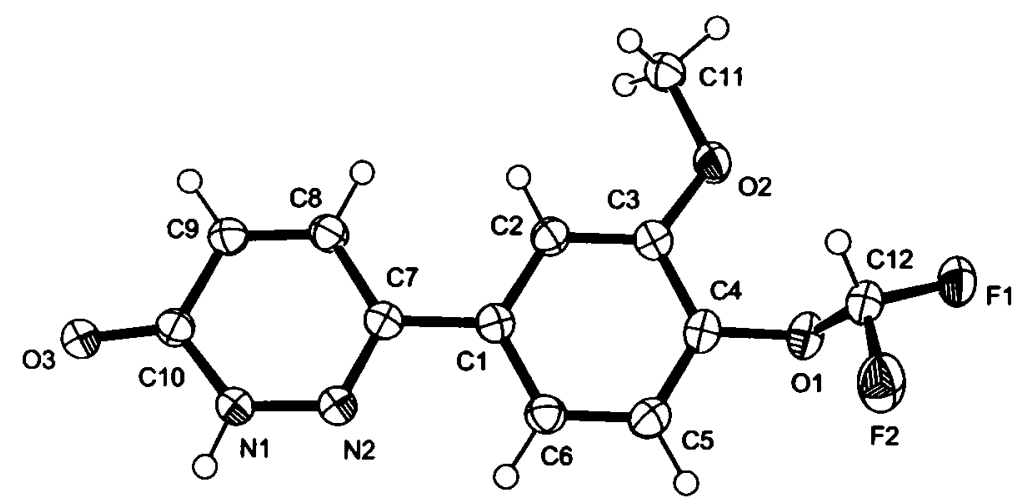

\begin{abstract}
$\mathrm{C}_{12} \mathrm{H}_{10} \mathrm{~F}_{2} \mathrm{~N}_{2} \mathrm{O}_{3}$, monoclinic, $P 121 / n 1$ (no. 14), $a=7.239(1) \AA, b=15.838(1) \AA, c=10.099(1) \AA$, $\beta=91.991(6)^{\circ}, V=1157.2 \AA^{3}, Z=4, R_{\mathrm{gt}}(F)=0.033$, $w R_{\text {ref }}\left(F^{2}\right)=0.093, T=109 \mathrm{~K}$.
\end{abstract}

\section{Source of material}

Zardaverine was purchased from SIGMA and crystallized by slow evaporation in methanol at room temperature.

\section{Discussion}

6-(4-difluoromethoxy-3-methoxyphenyl)-3(2H)-pyridazinone (zardaverine) selectively inhibits phosphodiesterase (PDE) isozymes III and IV [1], which regulate cyclic nucleotide levels in eukaryotic cells. The title compound, along with other dialkoxyphenol-containing compounds, have been examined as possible treatments for acute respiratory failure [1-3]. Zardaverine has also been used as a template compound to computationally generate a library of PDE4 inhibitors [4]. Two protein structures of zardaverine complexed with PDE4 type $D$ have been reported. One structure was solved to a resolution of $2.9 \AA$ [5] and the other was solved to $1.54 \AA$ [6]. In the higher resolution structure of PDE4D-zardaverine, the compound is found in three different conformations: $\mathrm{A}, \mathrm{B}$, and $\mathrm{C}$, with occupancies of $47 \%, 33 \%$, and $20 \%$, respectively. In all three conformers, the two rings are not co-planar. The torsion angles along $\mathrm{C} 6-\mathrm{C} 1-\mathrm{C} 7-\mathrm{N} 2$ are $39.6^{\circ}$ for $A,-146.6^{\circ}$ for $B$, and $-165.1^{\circ}$ for $C$. The $A$ and $B$ conformers are essentially equivalent except that the pyridazinone ring is flipped along the $\mathrm{Cl}-\mathrm{C} 7$ bond. This is possible because the pyridazinone ring is not anchored by $\mathrm{H}$ bonds to the protein.

In contrast, in the crystal structure reported here, the N1-H1 $\cdots$ $03(-x,-y+1,-z+1) H$-bond stabilizes the pyridazinone ring in a single conformation, and the crystal packing ensures that the two rings of the compound are essentially co-planar, with a $\mathrm{C} 6-\mathrm{C} 1-\mathrm{C} 7-\mathrm{N} 2$ torsion angle of $4.9(2)^{\circ}$. In the reported structure,

\footnotetext{
* Correspondence author (e-mail: wladek@iwonka.med.virginia.edu)
}

dimers of zardaverine are observed. The $\mathrm{H} 1$ atom (which is the only hydrogen in the structure capable of strong hydrogen bonding) forms a $\mathrm{H}$ bond with $\mathrm{O} 3(-x,-y+1,-z+1)$ of a symmetry-related molecule. This hydrogen bond is nearly ideal with an H1 $\cdots$ O3 distance of $1.887 \AA$ and an N1-H1 $\cdots \mathrm{O} 3$ angle of $174.04^{\circ}$. The N1-H1 …03 H-bonds are responsible for dimer formation. The molecules are packed in nearly planar layers. The $\mathrm{O} 3$ atom is also involved in a short contact interaction with hydrogen atom H12 $(-x+1,-y+1,-z+1)$, where the $03 \cdots H 12$ distance is $2.23(1) \AA$, and the $\mathrm{O} 3 \cdots \mathrm{H} 12-\mathrm{C12}(-x+1,-y+1,-z+1)$ angle is $163(1)^{\circ}$.

Table 1. Data collection and handling.

Crystal:

Wavelength:

$\mu$ :

Diffractometer, scan mode:

$2 \theta_{\max }$ :

$N(h k l)_{\text {measured, }} N(h k l)_{\text {unique: }}$

Criterion for $I_{\mathrm{obs}}, N(h k l)_{\mathrm{gt}}$ :

$N(\text { param })_{\text {refined: }}$

Programs:

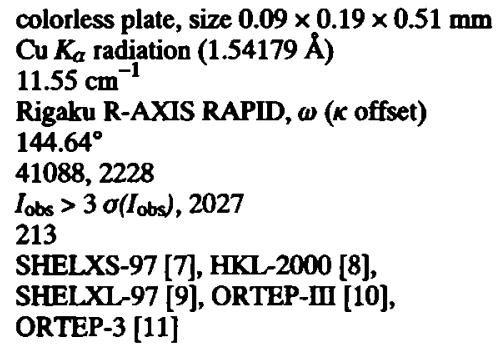

colorless plate, size $0.09 \times 0.19 \times 0.51 \mathrm{~mm}$ Cu $K_{a}$ radiation (1.54179 $\AA$ ) $11.55 \mathrm{~cm}^{-1}$

Rigaku R-AXIS RAPID, $\omega$ ( $\kappa$ offset) $144.64^{\circ}$

41088,2228

$I_{\mathrm{obs}}>3 \sigma\left(I_{\mathrm{obs}}\right), 2027$

213

SHELXS-97 [7], HKL-2000 [8], SHELXI-97 [9], ORTEP-III [10], ORTEP-3 [11]

Table 2. Atomic coordinates and displacement parameters (in $\AA^{2}$ ).

\begin{tabular}{llllll}
\hline Atom & Site & $x$ & $y$ & $z$ & $U_{\text {iso }}$ \\
\hline H(2) & $4 e$ & $0.216(2)$ & $0.4578(9)$ & $0.617(1)$ & $0.036(4)$ \\
H(12) & $4 e$ & $0.246(2)$ & $0.6737(9)$ & $0.920(1)$ & $0.030(3)$ \\
H(8) & $4 e$ & $0.293(2)$ & $0.3838(8)$ & $0.453(1)$ & $0.033(3)$ \\
H(9) & $4 e$ & $0.401(2)$ & $0.3170(9)$ & $0.265(1)$ & $0.036(4)$ \\
H(11A) & $4 e$ & $0.232(2)$ & $0.4199(9)$ & $0.844(1)$ & $0.039(4)$ \\
H(6) & $4 e$ & $0.233(2)$ & $0.6709(9)$ & $0.391(1)$ & $0.033(3)$ \\
H(1) & $4 e$ & $0.432(2)$ & $0.541(1)$ & $0.094(2)$ & $0.047(4)$ \\
H(11B) & $4 e$ & $0.016(2)$ & $0.4099(9)$ & $0.788(1)$ & $0.036(4)$ \\
H(5) & $4 e$ & $0.113(2)$ & $0.749(1)$ & $0.571(2)$ & $0.040(4)$ \\
H(11C) & $4 e$ & $0.066(2)$ & $0.4270(9)$ & $0.939(2)$ & $0.039(4)$ \\
\hline
\end{tabular}


Table 3. Atomic coordinates and displacement parameters (in $\AA^{2}$ ).

\begin{tabular}{|c|c|c|c|c|c|c|c|c|c|c|}
\hline Atom & Site & $x$ & $y$ & $z$ & $U_{11}$ & $U_{22}$ & $U_{33}$ & $U_{12}$ & $U_{13}$ & $U_{23}$ \\
\hline $\mathbf{F}(1)$ & $4 e$ & $0.0576(1)$ & $0.73471(5)$ & $1.00656(7)$ & $0.0499(5)$ & $0.0377(4)$ & $0.0321(4)$ & $0.0022(3)$ & $0.0099(3)$ & $-0.0072(3)$ \\
\hline$O(3)$ & $4 e$ & $0.4838(1)$ & $0.39220(5)$ & $0.04644(8)$ & $0.0359(5)$ & $0.0291(4)$ & $0.0255(4)$ & $0.0026(3)$ & $0.0046(3)$ & $0.0006(3)$ \\
\hline$O(1)$ & $4 e$ & $0.0261(1)$ & $0.69371(5)$ & $0.80161(8)$ & $0.0303(5)$ & $0.0331(4)$ & $0.0335(4)$ & $0.0027(3)$ & $0.0026(3)$ & $-0.0090(3)$ \\
\hline$F(2)$ & $4 e$ & $0.2375(1)$ & $0.78922(5)$ & $0.86334(8)$ & $0.0594(6)$ & $0.0403(4)$ & $0.0458(5)$ & $-0.0181(4)$ & $0.0119(4)$ & $-0.0108(3)$ \\
\hline$c(10)$ & $4 e$ & $0.4270(2)$ & $0.42321(7)$ & $0.1515(1)$ & $0.0263(6)$ & $0.0269(5)$ & $0.0251(5)$ & $0.0005(4)$ & $-0.0004(4)$ & $0.0003(4)$ \\
\hline$N(1)$ & $4 e$ & $0.4039(1)$ & $0.50838(6)$ & $0.16300(9)$ & $0.0328(6)$ & $0.0263(5)$ & $0.0242(5)$ & $0.0013(4)$ & $0.0045(4)$ & $0.0019(4)$ \\
\hline$c(6)$ & $4 e$ & $0.2044(2)$ & $0.64256(7)$ & $0.4752(1)$ & $0.0321(7)$ & $0.0265(5)$ & $0.0277(5)$ & $-0.0020(4)$ & $-0.0003(5)$ & $0.0012(4)$ \\
\hline$C(5)$ & $4 e$ & $0.1380(2)$ & $0.68855(7)$ & $0.5803(1)$ & $0.0317(7)$ & $0.0257(5)$ & $0.0341(6)$ & $0.0005(4)$ & $-0.0009(5)$ & $-0.0014(4)$ \\
\hline$C(9)$ & $4 e$ & $0.3826(2)$ & $0.37616(7)$ & $0.2677(1)$ & $0.0304(6)$ & $0.0246(5)$ & $0.0279(6)$ & $-0.0006(4)$ & $0.0014(4)$ & $0.0017(4)$ \\
\hline$O(2)$ & $4 e$ & $0.0896(1)$ & $0.52886(5)$ & $0.83307(7)$ & $0.0425(5)$ & $0.0293(4)$ & $0.0266(4)$ & $-0.0019(3)$ & $0.0081(3)$ & $-0.0010(3)$ \\
\hline C(1) & $4 e$ & $0.2358(2)$ & $0.55583(7)$ & $0.4879(1)$ & $0.0233(6)$ & $0.0267(5)$ & $0.0258(5)$ & $-0.0020(4)$ & $-0.0016(4)$ & $-0.0013(4)$ \\
\hline$C(2)$ & $4 e$ & $0.1988(2)$ & $0.51574(7)$ & $0.6075(1)$ & $0.0270(6)$ & $0.0240(5)$ & $0.0273(5)$ & $-0.0018(4)$ & $0.0007(4)$ & $-0.0008(4)$ \\
\hline$N(2)$ & $4 e$ & $0.3454(1)$ & $0.55121(6)$ & $0.26887(9)$ & $0.0301(6)$ & $0.0270(5)$ & $0.0250(5)$ & $0.0006(4)$ & $0.0022(4)$ & $0.0003(3)$ \\
\hline$C(8)$ & $4 e$ & $0.3239(2)$ & $0.41713(7)$ & $0.3756(1)$ & $0.0306(6)$ & $0.0267(5)$ & $0.0246(5)$ & $-0.0016(4)$ & $0.0012(4)$ & $0.0016(4)$ \\
\hline$c(3)$ & $4 e$ & $0.1318(2)$ & $0.56142(7)$ & $0.7131(1)$ & $0.0250(6)$ & $0.0287(5)$ & $0.0263(5)$ & $-0.0021(4)$ & $0.0010(4)$ & $-0.0006(4)$ \\
\hline$c(7)$ & $4 e$ & $0.3047(2)$ & $0.50708(7)$ & $0.3739(1)$ & $0.0229(6)$ & $0.0268(5)$ & $0.0246(5)$ & $-0.0017(4)$ & $-0.0011(4)$ & $0.0008(4)$ \\
\hline$C(4)$ & $4 e$ & $0.1025(2)$ & $0.64826(7)$ & $0.6975(1)$ & $0.0256(6)$ & $0.0290(5)$ & $0.0301(6)$ & $0.0000(4)$ & $0.0017(4)$ & $-0.0062(4)$ \\
\hline$C(12)$ & $4 e$ & $0.1526(2)$ & $0.71621(7)$ & $0.8979(1)$ & $0.0374(7)$ & $0.0279(6)$ & $0.0295(6)$ & $0.0017(5)$ & $0.0035(5)$ & $-0.0054(4)$ \\
\hline C(11) & $4 e$ & $0.1044(2)$ & $0.43886(7)$ & $0.8503(1)$ & $0.0376(8)$ & $0.0300(6)$ & $0.0283(6)$ & $-0.0018(5)$ & $0.0029(5)$ & $0.0025(4)$ \\
\hline
\end{tabular}

Acknowledgments. This work was supported by contract GI11496 from HKL Research, Inc. The authors would like to thank Rigaku/MSC for the loan of the R-AXIS RAPID diffractometer.

\section{References}

1. Rabe, K. F.; Tenor, H.; Dent, G; Schudt, C.; Nakashima, M.; Magnussen, H.: Identification of PDE isozymes in human pulmonary artery and effect of selective PDE inhibitors. Am. J. Physiol. 266 (1994) L536-L543.

2. Schmidt, D. T.; Watson, N.; Dent, G.; Ruhlmann, E.; Branscheid, D.; Magnussen, H.; Rabe, K. F.: The effect of selective and non-selective phosphodiesterase inhibitors on allergen- and leukotriene $C(4)$-induced contractions in passively sensitized human airways. Brit. J. Pharmacol. 131 (2000) 1607-1618.

3. Schermuly, R. T.; Leuchte, H.; Ghofrani, H. A.; Weissmann, N.; Rose, F.; Kohstall, M.; Olschewski, H.; Schudt, C.; Grimminger, F.; Seeger, W.; Walmrath, D.: Zardaverine and aerosolised iloprost in a model of acute respiratory failure. Eur. Respir. J. 22 (2003) 342-347.

4. Krier, M.; Araújo-Júnior, J. X.; Schmitt, M.; Duranton, J.; JustianoBasaran, H.; Lugnier, C.; Bourguignon, J.; Rognan, D.: Design of smallsized libraries by combinatorial assembly of linkers and functional groups to a given scaffold: application to the structure-based optimization of a phosphodiesterase 4 inhibitor. J. Med. Chem. 48 (2005) 3816-3822.

5. Lee, M. E.; Markowitz, J.; Lee, J.; Lee, H.: Crystal structure of phosphodiesterase 4D and inhibitor complex (1). FEBS Lett. 530 (2002) 53-58.
6. Card, G. L.; England, B. P.; Suzuki, Y.; Fong, D.; Powell, B.; Lee, B.; Luu, C.; Tabrizizad, M.; Gillette, S.; Ibrahim, P. N.; Artis, D. R.; Bollag, G.; Milbum, M. V.; Kim, S.; Schlessinger, J.; Zhang, K. Y. J.: Structural basis for the activity of drugs that inhibit phosphodiesterases. Structure 12 (2004) 2233-2247.

7. Sheldrick, G. M.: SHELXS-97. Program for the Solution of Crystal Structures. University of Göttingen, Germany 1997.

8. Otwinowski, Z; Minor, W.: Processing of X-ray diffraction data collected in oscillation mode. Methods Enzymol. 276 (1997) 307-326.

9. Sheldrick, G. M.: SHELXI-97. Program for the Refinement of Crystal Structures. University of Göttingen, Germany 1997.

10. Bumett, M. N.; Johnson, C. K.: ORTEP-III. Oak Ridge Thermal Ellipsoid Plot Program for Crystal Structure Illustrations. Report ORNL-6895, Oak Ridge National Laboratory, Tennessee, USA 1996.

11. Farrugia, L. J.: ORTEP-3 for Windows - a version of ORTEP-III with a Graphical User Interface (GUT). J. Appl. Crystallogr. 30 (1997) 565. 